\title{
Anomaly matching for the phase diagram of massless $\mathbb{Z}_{N}$-QCD
}

\author{
Yuya Tanizaki, ${ }^{1, *}$ Yuta Kikuchi, ${ }^{2,3, \dagger}$ Tatsuhiro Misumi, ${ }^{4,5,6, \$}$ and Norisuke Sakai ${ }^{5,6,8}$ \\ ${ }^{1}$ RIKEN BNL Research Center, Brookhaven National Laboratory, Upton, New York 11973, USA \\ ${ }^{2}$ Department of Physics, Kyoto University, Kyoto 606-8502, Japan \\ ${ }^{3}$ Department of Physics and Astronomy, Stony Brook University, Stony Brook, New York 11794-3800, USA \\ ${ }^{4}$ Department of Mathematical Science, Akita University, Akita 010-8502, Japan \\ ${ }^{5}$ Research and Education Center for Natural Sciences, Keio University, Kanagawa 223-8521, Japan \\ ${ }^{6}$ iTHEMS, RIKEN, Wako, Saitama 351-0198, Japan
}

(Received 4 December 2017; published 15 March 2018)

\begin{abstract}
We elucidate that the phase diagram of massless $N$-flavor QCD under a $\mathbb{Z}_{N}$ flavor-twisted boundary condition (massless $\mathbb{Z}_{N}$-QCD) is constrained by a 't Hooft anomaly involving two-form gauge fields. As a consequence, massless $\mathbb{Z}_{N}$-QCD turns out to realize persistent order at any temperature and quark chemical potential; namely, the symmetric and gapped phase is strictly forbidden. This is the first result on the finite$(T, \mu)$ phase diagram in QCD-type theories based on anomaly matching related to center and discrete axial symmetries.
\end{abstract}

DOI: $10.1103 /$ PhysRevD.97.054012

\section{INTRODUCTION}

Conventional wisdom of classical statistical physics tells us that different phases of matter are distinguished by different patterns of spontaneous symmetry breaking (SSB). This idea develops Ginzburg-Landau effective theory $[1,2]$ and gives a useful guideline to understand the nature of types of matters. For quantum field theories (QFTs), however, the Ginzburg-Landau description is not always a good starting point, especially when QFTs have a 't Hooft anomaly [3-5]. This is because the anomaly matching argument rules out the symmetric and gapped phase, which we call the trivial phase. The necessity of a beyond-Ginzburg-Landau description has also been clarified, for instance, by Haldane conjecture [6-10], deconfined quantum criticality [11-13], in condensed matter physics. Recent developments about QFTs suggest that the absence of a trivial phase is closely related to the existence of a 't Hooft anomaly, and many quantum systems have been reconsidered from this viewpoint [14-36].

In this paper, we claim that quantum chromodynamics (QCD) should also be added to the list of quantum systems that require the beyond-Ginzburg-Landau treatment. QCD

\footnotetext{
yuya.tanizaki@riken.jp

kikuchi@ruby.scphys.kyoto-u.ac.jp

*misumi@phys.akita-u.ac.jp

§norisuke.sakai@gmail.com
}

Published by the American Physical Society under the terms of the Creative Commons Attribution 4.0 International license. Further distribution of this work must maintain attribution to the author(s) and the published article's title, journal citation, and DOI. Funded by SCOAP ${ }^{3}$. is the fundamental law of strong interaction; thus it can provide the first-principle calculation of finite-density nuclear matters, which is an important subject when trying to understand, for instance, the interior of neutron stars [37-39]. Because of the notorious sign problem, however, no $a b$ initio lattice QCD simulation is yet available at finite quark chemical potentials [40-44].

We consider massless $N$-flavor QCD with color $N$ in four dimensions, which has the vectorlike continuous symmetry and discrete axial symmetry, and we show by an explicit calculation that there is a mixed 't Hooft anomaly between those symmetries. Imposing the flavortwisted boundary condition in the compactified direction so that the $\mathbb{Z}_{N}$ center symmetry appears (known as $\mathbb{Z}_{N}$-QCD $[33,45-54])$, the above mixed anomaly turns out to survive at any temperature and quark chemical potential. Anomaly matching precludes the existence of the trivial phase for massless $\mathbb{Z}_{N}$-QCD. We discuss the consistency of our result with previous studies and argue its implications for the phase diagram of massless $\mathbb{Z}_{N}$-QCD.

\section{ANOMALY MATCHING OF MASSLESS $\mathbb{Z}_{N}$-QCD}

We first compute a 't Hooft anomaly of massless $N$-flavor QCD in four dimensions, and we derive the anomaly of massless $\mathbb{Z}_{N}$-QCD using the technique given in [33].

\section{A. Massless $\boldsymbol{N}$-flavor QCD and 't Hooft anomaly}

We consider four-dimensional $S U(N)$ Yang-Mills theory with $N$ massless Dirac fermions in the fundamental representation, i.e., massless QCD with $N_{\mathrm{c}}=N_{\mathrm{f}}=N$. 
We discriminate between the color and flavor $S U(N)$ groups by denoting them as $S U(N)_{\text {c }}$ and $S U(N)_{\mathrm{f}}$, respectively. The $S U(N)$ c gauge field is denoted as $a$, and the field strength is given by $G_{\mathrm{c}}=\mathrm{d} a+\mathrm{i} a \wedge a$. We represent the Dirac field $q=\left(q_{c f}\right)_{c, f=1, \ldots, N}$ as an $N \times N$ matrixvalued Grassmannian variable, on which $S U(N)_{\mathrm{c}} \times$ $S U(N)_{\mathrm{f}}$ acts as a bifundamental representation: $q \mapsto$ $U_{\mathrm{c}} q U_{\mathrm{f}}^{\dagger}$ for $\left(U_{\mathrm{c}}, U_{\mathrm{f}}\right) \in S U(N) \mathrm{c} \times S U(N)_{\mathrm{f}}$. The classical action of this theory is given by

$S=\frac{1}{2 g^{2}} \int \operatorname{tr}_{\mathrm{c}}\left(G_{\mathrm{c}} \wedge * G_{\mathrm{c}}\right)+\int \mathrm{d}^{4} x \operatorname{tr}_{\mathrm{f}}\left\{\bar{q} \gamma_{\mu} D_{\mu}(a) q\right\}$,

where $\operatorname{tr}_{\mathrm{c}}$ and $\operatorname{tr}_{\mathrm{f}}$ denote the trace operation in color and flavor spaces, respectively, and $D(a)=\mathrm{d}+\mathrm{i} a$ is the covariant derivative.

We pay attention to the vectorlike symmetry,

$$
\frac{S U(N)_{\mathrm{f}} \times U(1)_{\mathrm{q}}}{\left(\mathbb{Z}_{N}\right)_{\mathrm{c}-\mathrm{q}}\left(\mathbb{Z}_{N}\right)_{\mathrm{f}-\mathrm{q}}}
$$

and the anomaly free subgroup of $U(1)$ axial symmetry, $\left(\mathbb{Z}_{2 N}\right)_{\text {axial }}$. We shall explain the details of these symmetries.

The quark field $q$ is in the representation of vectorlike symmetry $\quad S U(N)_{\mathrm{c}} \times S U(N)_{\mathrm{f}} \times U(1)_{\mathrm{q}}, \quad q \mapsto \mathrm{e}^{\mathrm{i} \alpha} U_{\mathrm{c}} q U_{\mathrm{f}}^{\dagger}$ for $\left(U_{\mathrm{c}}, U_{\mathrm{f}}, \mathrm{e}^{\mathrm{i} \alpha}\right) \in S U(N)_{\mathrm{c}} \times S U(N)_{\mathrm{f}} \times U(1)_{\mathrm{q}}$. However, there are two $\mathbb{Z}_{N}$ subgroups that do not change $q$. One is generated by $\left(\omega, 1, \omega^{-1}\right) \in S U(N)_{\mathrm{c}} \times S U(N)_{\mathrm{f}} \times U(1)_{\mathrm{q}}$ $\left(\omega=\mathrm{e}^{2 \pi \mathrm{i} / N}\right)$, and we denote it as $\left(\mathbb{Z}_{N}\right)_{\mathrm{c}-\mathrm{q}}$. The other is generated by $(1, \omega, \omega) \in S U(N)_{\mathrm{c}} \times S U(N)_{\mathrm{f}} \times U(1)_{\mathrm{q}}$, and we denote it as $\left(\mathbb{Z}_{N}\right)_{\mathrm{f}-\mathrm{q}}$. We thus regard (2) as a symmetry with faithful representations.

For massless quark field $q$, there is the axial $U(1)$ symmetry, $q \mapsto \mathrm{e}^{\mathrm{i} \alpha \gamma_{5}} q$, for the classical action (1), but the fermion functional integration measure $\mathcal{D} \bar{q} \mathcal{D} q$ is changed as $\mathcal{D} \bar{q} \mathcal{D} q \mapsto \mathcal{D} \bar{q} \mathcal{D} q \exp \left(\mathrm{i} 2 \alpha \frac{N}{8 \pi^{2}} \int \operatorname{tr}_{\mathrm{c}}\left(G_{c} \wedge G_{c}\right)\right) \quad[55,56]$. Only when $\alpha$ is quantized to $2 \pi /(2 N)$ does this transformation become the symmetry of $N$-flavor massless QCD. This is the $\left(Z_{2 N}\right)_{\text {axial }}$ symmetry.

In order to detect the mixed 't Hooft anomaly between the vectorlike symmetry (2) and $\left(\mathbb{Z}_{2 N}\right)_{\text {axial }}$, we introduce the background gauge fields for the vectorlike symmetry. Background gauge fields consist of the following ingredients:

(i) $S U(N)_{\mathrm{f}}$ one-form gauge field: $A_{\mathrm{f}}$

(ii) $U(1)_{\mathrm{q}}$ one-form gauge field: $A_{\mathrm{q}}$

(iii) $\left(\mathbb{Z}_{N}\right)_{\mathrm{c}-\mathrm{q}}$ two-form gauge field: $B_{\mathrm{c}}\left(N B_{\mathrm{c}}=\mathrm{d} C_{\mathrm{c}}\right)$

(iv) $\left(\mathbb{Z}_{N}\right)_{\mathrm{f}-\mathrm{q}}$ two-form gauge field: $B_{\mathrm{f}}\left(N B_{\mathrm{f}}=\mathrm{d} C_{\mathrm{f}}\right)$

The $\mathbb{Z}_{N}$ two-form gauge field $B$ may require an explanation: we realize it as a pair of $U(1)$ two-form gauge field $B$ and $U(1)$ one-form gauge field $C$ with the constraint $N B=\mathrm{d} C$. It generates a $\mathbb{Z}_{N}$ one-form symmetry transformation acting on Wilson line operators [57], which arises after gauging $S U(N)_{\mathrm{f}} \times U(1)_{\mathrm{q}} \quad[24,28,32,33]$. Introducing the two-form gauge fields $B_{\mathrm{c}}$ and $B_{\mathrm{f}}$, the $S U(N)_{\text {c.f }}$ gauge connections are once promoted to $U(N)$ gauge connections [58],

$$
\tilde{a}=a+\frac{1}{N} C_{\mathrm{c}}, \quad \tilde{A_{\mathrm{f}}}=A_{\mathrm{f}}+\frac{1}{N} C_{\mathrm{f}} .
$$

The covariant derivative is replaced as

$$
D\left(\tilde{a}, \widetilde{A_{\mathrm{f}}}, A_{\mathrm{q}}\right) q=\left(\mathrm{d}+\mathrm{i} \tilde{a}-\mathrm{i}\left(\widetilde{A_{\mathrm{f}}}\right)^{t}+\mathrm{i} A_{\mathrm{q}}\right) q,
$$

by the minimal coupling procedure. More explicitly,

$$
\left(D_{\mu} q\right)_{c f}=\left(\partial_{\mu}+\mathrm{i}\left(A_{\mathrm{q}}\right)_{\mu}\right) q_{c f}+\mathrm{i} \tilde{a}_{\mu, c c^{\prime}} q_{c^{\prime} f}-\mathrm{i} q_{c f^{\prime}}\left(\widetilde{A_{\mathrm{f}}}\right)_{\mu, f^{\prime} f} .
$$

(4) must be regarded as a shorthand notation of (5), where the repeated indices are summed.

With this setup, we consider the partition function under these background gauge fields, $\mathcal{Z}\left[\left(A_{\mathrm{f}}, A_{\mathrm{q}}, B_{\mathrm{c}}, B_{\mathrm{f}}\right)\right]$. Applying the $\left(\mathbb{Z}_{2 N}\right)_{\text {axial }}$ transformation, we can compute the change of the partition function,

$$
\mathcal{Z}\left[\left(A_{\mathrm{f}}, A_{\mathrm{q}}, B_{\mathrm{c}}, B_{\mathrm{f}}\right)\right] \mapsto \mathcal{Z}\left[\left(A_{\mathrm{f}}, A_{\mathrm{q}}, B_{\mathrm{c}}, B_{\mathrm{f}}\right)\right] \exp (\mathrm{i} \mathcal{A}) .
$$

The 't Hooft anomaly $\mathcal{A}$ again comes from the change of the fermion measure $\mathcal{D} \bar{q} \mathcal{D} q$, and the Fujikawa method gives

$$
\mathcal{A}=2 \frac{2 \pi}{2 N} \frac{1}{8 \pi^{2}} \int \operatorname{tr}_{\mathrm{c}, \mathrm{f}}[F \wedge F],
$$

with $F=\mathrm{i}^{-1} D\left(\tilde{a}, \tilde{A_{\mathrm{f}}}, A_{\mathrm{q}}\right) \wedge D\left(\tilde{a}, \tilde{A_{\mathrm{f}}}, A_{\mathrm{q}}\right) \cdot \operatorname{tr}_{\mathrm{c}, \mathrm{f}}$ denotes the trace operation over both color and flavor spaces. The explicit computation shows that

$$
F=(\mathrm{d} \tilde{a}+\mathrm{i} \tilde{a} \wedge \tilde{a})-\left(\mathrm{d} \widetilde{A_{\mathrm{f}}}+\mathrm{i} \widetilde{A_{\mathrm{f}}} \wedge \widetilde{A_{\mathrm{f}}}\right)^{t}+\mathrm{d} A_{\mathrm{q}} .
$$

We obtain from (7) and (8) that (modulo $2 \pi$ )

$$
\mathcal{A}=-\frac{N}{2 \pi} \int B_{\mathrm{c}} \wedge B_{\mathrm{f}}
$$

Since $\mathcal{A}$ is nontrivial, there is a mixed 't Hooft anomaly between the vectorlike symmetry (2) and $\left(\mathbb{Z}_{2 N}\right)_{\text {axial }}$ for four-dimensional massless $N$-flavor QCD.

\section{B. Massless $\mathbb{Z}_{N}$-QCD and 't Hooft anomaly}

We perform the circle compactification of size $L$ and put the boundary condition on the quark field as

$$
q\left(\boldsymbol{x}, x_{4}+L\right)=q\left(\boldsymbol{x}, x_{4}\right) \Omega,
$$


where $\left(\omega=\mathrm{e}^{2 \pi \mathrm{i} / N},-\pi<\phi \leq \pi\right.$, and $\left.\mu \in \mathbb{R}\right)$

$$
\Omega=\operatorname{diag}\left[1, \omega, \ldots, \omega^{N-1}\right] \mathrm{e}^{\mathrm{i} \phi+\mu L} .
$$

Here, $\mu$ is the quark chemical potential. This theory is called $\mathbb{Z}_{N}$-QCD, and we denote its partition function as $\mathcal{Z}_{\Omega}$ [45-54]. Although the twisted boundary condition is imposed, we call $T \equiv L^{-1}$ a temperature of massless $\mathbb{Z}_{N}$-QCD. By performing the gauge transformation, we can express this symmetry-twisted partition function as

$$
\mathcal{Z}_{\Omega}=\operatorname{tr}_{\mathcal{H}}\left[\mathrm{e}^{-L(\hat{H}-\mu \hat{Q})} \exp \left(\mathrm{i} \sum_{f=1}^{N} \frac{2 \pi f}{N} \hat{Q}_{f}\right)\right],
$$

where $\hat{H}$ is the QCD Hamiltonian, $\mathcal{H}$ is the QCD Hilbert space, $\hat{Q}_{f}=\int \mathrm{d}^{3} \boldsymbol{x} \hat{q}_{f}^{\dagger} \hat{q}_{f}(\boldsymbol{x})$ is the quark number of flavor $f$, and $\hat{Q}=\sum_{f} \hat{Q}_{f}$ is the total quark number (for simplicity of the expression, we set $\phi=\pi$ ). This twisted boundary condition plays an essential role in the existence of the 't Hooft anomaly in the $S^{1}$-compactified theory [33]. The same boundary condition is also important for the large- $N$ volume independence of the $\mathbb{C} P^{N-1}$ model [33,59-63].

We first describe the symmetry of massless $\mathbb{Z}_{N}$-QCD as three-dimensional QFT. There are three symmetries of importance, $\left(\mathbb{Z}_{N}\right)_{\text {shift,c-q }}, \quad\left(U(1)_{\mathrm{f}}^{N-1} \times U(1)_{\mathrm{q}}\right) /\left(\left(\mathbb{Z}_{N}\right)_{\mathrm{c}-\mathrm{q}} \times\right.$ $\left.\left(\mathbb{Z}_{N}\right)_{\mathrm{f}-\mathrm{q}}\right)$, and $\left(\mathbb{Z}_{2 N}\right)_{\text {axial }}$. To explain $\left(\mathbb{Z}_{N}\right)_{\text {shift,c-q }}$, it is better to regard $\Omega$ as the background holonomy of vectorlike symmetry along the compactified direction,

$$
\Omega=\left\{\mathcal{P} \exp \left(\mathrm{i} \int_{0}^{L} A_{\mathrm{f}}\right)\right\}^{-1} \exp \left(\mathrm{i} \int_{0}^{L} A_{\mathrm{q}}\right) .
$$

We consider the $\left(\mathbb{Z}_{N}\right)_{\text {shift }}$ transformation defined by

$q=\left(q_{c, 1}, \ldots, q_{c, N}\right) \mapsto\left(q_{c, 2}, \ldots, q_{c, N}, q_{c, 1}\right)=: q S^{-1}$.

Under this transformation, the boundary condition (10) is changed as

$$
\Omega \mapsto S \Omega S^{-1}=\omega \Omega
$$

To maintain the boundary condition, we combine the $\left(\mathbb{Z}_{N}\right)_{\text {c-q }}$ zero-form and $\left(\mathbb{Z}_{N}\right)_{\text {shift }}$ transformations, and we define the $\left(\mathbb{Z}_{N}\right)_{\text {shift,c-q }}$ symmetry $[33,54]$,

$$
\operatorname{tr}_{\mathrm{c}}(\Phi) \mapsto \omega \operatorname{tr}_{\mathrm{c}}(\Phi), \quad q \mapsto q S^{-1},
$$

where $\Phi:=\mathcal{P} \exp \left(\mathrm{i} \int_{S^{1}} a\right)$ is the color Polyakov loop. The vectorlike symmetry must commute with the matrix $\Omega$ defining the boundary condition, and the symmetry (2) is explicitly broken to its maximal Abelian subgroup, $\left(U(1)_{\mathrm{f}}^{N-1} \times U(1)_{\mathrm{q}}\right) /\left(\left(\mathbb{Z}_{N}\right)_{\mathrm{c}-\mathrm{q}} \times\left(\mathbb{Z}_{N}\right)_{\mathrm{f}-\mathrm{q}}\right)$.
Introducing the three-dimensional background gauge fields for $\left(\mathbb{Z}_{N}\right)_{\text {shift,c-q }}$ and $\left(U(1)_{\mathrm{f}}^{N-1} \times U(1)_{\mathrm{q}}\right) /\left(\left(\mathbb{Z}_{N}\right)_{\mathrm{c}-\mathrm{q}} \times\right.$ $\left.\left(\mathbb{Z}_{N}\right)_{\mathrm{f}-\mathrm{q}}\right)$, we obtain the following two-form gauge fields by use of the four-dimensional language [33],

$$
B_{\mathrm{c}}=B_{\mathrm{c}}^{(1)} \wedge L^{-1} \mathrm{~d} x^{4}+B_{\mathrm{c}}^{(2)}, \quad B_{\mathrm{f}}=B_{\mathrm{f}}^{(2)} .
$$

Here, $B_{\mathrm{c}}^{(1)}$ is a one-form gauge field for $\left(\mathbb{Z}_{N}\right)_{\text {shift,c-q }}$ zeroform symmetry in three dimensions, and $B_{\mathrm{c}, \mathrm{f}}^{(2)}$ are two-form gauge fields for $\left(\mathbb{Z}_{N}\right)_{c-q, f-q}$ one-form symmetries, respectively, also in three dimensions. Substituting it into the fourdimensional anomaly (9), we obtain the anomaly for massless $\mathbb{Z}_{N}$-QCD:

$$
\mathcal{A}=-\frac{N}{2 \pi} \int B_{\mathrm{c}}^{(1)} \wedge B_{\mathrm{f}}^{(2)} \in \frac{2 \pi}{N} \mathbb{Z} .
$$

This gives the mixed 't Hooft anomaly of massless $\mathbb{Z}_{N^{-}}$ QCD among $\left(\mathbb{Z}_{N}\right)_{\text {shift,c-q }},\left(U(1)_{\mathrm{f}}^{N-1} \times U(1)_{\mathrm{q}}\right) /\left(\left(\mathbb{Z}_{N}\right)_{\mathrm{c}-\mathrm{q}} \times\right.$ $\left.\left(\mathbb{Z}_{N}\right)_{\text {f-q }}\right)$, and $\left(\mathbb{Z}_{2 N}\right)_{\text {axial }}$, for any $N \geq 2$. To match the anomaly, the phase diagram of massless $\mathbb{Z}_{N^{-}}$QCD must realize the persistent order at any temperature $L^{-1}$ and quark chemical potential $\mu$ : the trivial phase is strictly excluded by the anomaly-matching condition.

\section{PHASE STRUCTURE OF MASSLESS $\mathbb{Z}_{N}$-QCD}

In certain limits of $T=L^{-1}$ and $\mu$, the phase structure of massless $\mathbb{Z}_{N}$-QCD is calculable, and thus we can check how the anomaly matching is satisfied in those limits. We consider high temperature or large chemical potential as a limit where the reliable perturbative calculations are available. We also note that the lattice simulation [50] suggests that at the large- $L$ and small $-\mu$ region, the anomaly (18) is matched by the SSB of $\left(\mathbb{Z}_{2 N}\right)_{\text {axial }} \rightarrow \mathbb{Z}_{2}$. Since this naturally breaks the continuous chiral symmetry by developing quark bilinear condensate $\langle\bar{q} q\rangle$, this is also consistent with a 't Hooft anomaly of continuous chiral symmetry [3] at $L=\infty$ and $\mu=0$.

\section{A. High temperature limit}

At small $L$ and $\mu=0$, we compute the one-loop effective potential of the color Polyakov loop $\Phi[64,65]$. The gluon contribution is

$$
V_{\mathrm{gl}}(\Phi)=-\frac{2}{\pi^{2} L^{4}} \sum_{n \geq 1} \frac{1}{n^{4}}\left(\left|\operatorname{tr}_{\mathrm{c}}\left(\Phi^{n}\right)\right|^{2}-1\right) .
$$

The quark contribution (at $\mu=0$ ) is

$$
V_{\mathrm{qk}}(\Phi)=\frac{2 N^{-3}}{\pi^{2} L^{4}} \sum_{n \geq 1} \frac{1}{n^{4}}\left(\mathrm{e}^{\mathrm{i} n N \phi} \operatorname{tr}_{\mathrm{c}}\left(\Phi^{N n}\right)+\text { H.c. }\right) .
$$


The gluon contribution is $V_{\mathrm{gl}}=O\left(N^{2}\right)$, while the quark contribution is $V_{\mathrm{qk}}=O\left(N^{-2}\right)$ [54]. Therefore, the hightemperature behavior of $\mathbb{Z}_{N}$-QCD is essentially determined by the gluonic contribution. $\left(\mathbb{Z}_{N}\right)_{\text {shift,c-q }}$ is spontaneously broken, and the vacuum is $\Phi \propto \mathbf{1}_{N}$. The 't Hooft anomaly (18) is matched by the SSB of $\left(\mathbb{Z}_{N}\right)_{\text {shift,c-q. }}$. Strictly speaking, this perturbative argument is subtle because the threedimensional effective theory is a strongly coupled gauge theory, but the above observation is consistent with lattice simulation [50].

This argument at high temperatures is valid at any $U(1)$ phase $\phi$ in $\Omega$, but we would like to comment that the $\left(\mathbb{Z}_{N}\right)_{\text {shift,c-q }}$ symmetry is enhanced to $\mathbb{Z}_{N} \rtimes \mathbb{Z}_{2}$ when $\phi$ is quantized to $\pi / N$, although this does not give new anomalies. When the flavor-independent boundary condition is set instead of the flavor-twisted boundary condition, no center symmetry exists at generic $\phi$, but a $\mathbb{Z}_{2}$ subgroup of $\mathbb{Z}_{N} \rtimes \mathbb{Z}_{2}$ becomes a symmetry when $\phi \in(\pi / N) \mathbb{Z}$, which derives a mixed 't Hooft anomaly with other symmetries when local counterterms cannot cancel it [28]. This gives an underlying reason for the Roberge-Weiss phase transition [66]. In our case, the SSB occurs as $\mathbb{Z}_{N} \rtimes \mathbb{Z}_{2} \rightarrow \mathbb{Z}_{2}$, which is consistent with the absence of new anomalies.

\section{B. High density limit}

In a large- $\mu$ limit, it is widely expected that color superconductivity appears [37]. In the following discussion, we set $N=3$. To discuss color superconductivity, we first prepare gauge "noninvariant" scalar and pseudoscalar diquark operators $(N=3)$,

$$
\begin{aligned}
& \Delta_{c_{1} f_{1}}=\varepsilon_{c_{1} c_{2} c_{3}} \varepsilon_{f_{1} f_{2} f_{3}}\left(q_{f_{2} c_{2}}^{t} \mathrm{i} \gamma_{0} \gamma_{2} \gamma_{5} q_{c_{3} f_{3}}\right), \\
& \Delta_{c_{1} f_{1}}^{\prime}=\varepsilon_{c_{1} c_{2} c_{3}} \varepsilon_{f_{1} f_{2} f_{3}}\left(q_{f_{2} c_{2}}^{t} \mathrm{i} \gamma_{0} \gamma_{2} q_{c_{3} f_{3}}\right) .
\end{aligned}
$$

Since these are not gauge invariant, this analysis makes sense only at weak coupling, but they are useful for Higgs phenomena. $\left(\mathbb{Z}_{2 N}\right)_{\text {axial }}$ rotates $\Delta$ and $\Delta^{\prime}$ by an angle $2 \pi / N$, and thus it is completely broken down to $\mathbb{Z}_{2}$ if $\Delta$ or $\Delta^{\prime}$ gets an expectation value. Below, we only use the scalar condensate $\Delta$ via an appropriate rotation of $\left(\mathbb{Z}_{2 N}\right)_{\text {axial }}$.

At sufficiently large $\mu$ and small temperatures, we expect to have $\Delta_{c f}=\Delta_{\mathrm{CFL}} \delta_{c f}$, which is called color-flavor locking (CFL) [37]. In this phase, the Higgs phenomenon with fundamental scalars occurs and all gluons become massive, which is why weak-coupling analysis is applicable. Let us combine it with the analysis of anomaly matching in order to speculate physics in the strongly coupled regime. For that purpose, it is important to identify the pattern of SSB by gauge-invariant order parameters [54]: $\Delta^{\dagger} \Delta \sim(\bar{q} \bar{q}) q q$ and $\varepsilon_{c_{1} c_{2} c_{3}} \Delta_{c_{1} f_{1}} \Delta_{c_{2} f_{2}} \Delta_{c_{3} f_{2}} \sim(q q)^{3}$. The latter clearly breaks $U(1)_{\mathrm{B}} \equiv U(1)_{\mathrm{q}} /\left(\mathbb{Z}_{N}\right)_{\mathrm{c}-\mathrm{q}} \rightarrow \mathbb{Z}_{2}$, which is the superfluid phase. As we have already discussed, the diquark condensate also breaks $\left(\mathbb{Z}_{2 N}\right)_{\text {axial }}$. Indeed, this is required by anomaly matching because we can obtain the anomaly (9) without gauging $U(1)_{\mathrm{q}}$ symmetry by setting $B_{\mathrm{c}}=B_{\mathrm{f}}$ when $N \geq 3$ [33]. In the CFL phase, $\left(\mathbb{Z}_{N}\right)_{\text {shift,c-q }}$ is unbroken. We remark that the anomaly is also consistent with another possibility of a CFL-like phase, where three diquark condensates have distinct values and $\left(\mathbb{Z}_{N}\right)_{\text {shift,c-q }}$ is broken [51].

It is also expected from the analysis of effective models [51] that the two-flavor pairing phase (2SC) or the u-flavor pairing phase (uSC) exist at large- $\mu$ regions. These phases are characterized by $\Delta_{c f}=\Delta_{2 \mathrm{SC}} \delta_{c, 3} \delta_{f, 3}$ and by $\Delta_{c f}=\Delta_{\mathrm{uSC}}\left(\delta_{c, 2} \delta_{f, 2}+\delta_{c, 3} \delta_{f, 3}\right)$, respectively. In the uSC phase, all gluons become massive, while there are $S U(2)$ massless gluons in the 2SC phase and perturbative computations are useless in the 2SC phase. In these phases, the $\left(\mathbb{Z}_{N}\right)_{\text {shift,c-q }}$ is spontaneously broken due to the asymmetric diquark condensates in addition to the breaking of $\left(\mathbb{Z}_{2 N}\right)_{\text {axial }}$, while $U(1)_{\mathrm{B}}$ is unbroken ${ }^{1}$ due to $(q q)^{3}=0$. This is again consistent with anomaly matching.

\section{Phase structure of massless $\mathbb{Z}_{\mathbf{3}}$-QCD}

In the known phases discussed above, $U(1)_{\mathrm{f}}^{N-1}$ is always unbroken. From the viewpoint of anomaly matching, there is no reason to rule out the SSB of $U(1)_{\mathrm{f}}^{N-1}$. Assuming that $U(1)_{\mathrm{f}}^{N-1}$ is unbroken everywhere, we can derive an inequality between the "deconfinement" temperature $T_{\text {deconf }}$, above which $\left(\mathbb{Z}_{N}\right)_{\text {shift,c-q }}$ is broken, and the chiral restoration temperature $T_{\text {chiral }}$, above which $\left(\mathbb{Z}_{2 N}\right)_{\text {axial }}$ is unbroken [23,26,28]: when $N \geq 3$, for any $\mu$,

$$
T_{\text {chiral }}(\mu) \geq T_{\text {deconf }}(\mu) .
$$

Under this assumption, the anomaly matching requires that the discrete axial symmetry $\left(\mathbb{Z}_{2 N}\right)_{\text {axial }}$ must be spontaneously broken at low temperatures, which automatically requires the SSB of continuous axial symmetry since $\left(\mathbb{Z}_{2 N}\right)_{\text {axial }} \subset S U(N)_{\text {left }} \times S U(N)_{\text {right }} \times U(1)_{\mathrm{q}}$.

We schematically show finite- $(T, \mu)$ phase diagrams of massless $\mathbb{Z}_{3}$-QCD in Fig. 1 . In the strongly coupled region, not much is known about massless $\mathbb{Z}_{N}$-QCD, and we present just two possible scenarios that are consistent with the anomaly. It is an interesting and testable question whether the inequality (22) at $\mu=0$ is saturated or not for lattice QCD simulation. It is notable that the symmetric and gapped phase cannot appear, although such phases were observed in the study based on the effective model [51]. The appearance of forbidden phases is generic when a Ginzburg-Landau-type effective approach is used for QFT with a 't Hooft anomaly.

\footnotetext{
${ }^{1}$ In the gauge-noninvariant argument, it corresponds to the simultaneous baryon and color rotation $U(1)_{\mathrm{B}+\mathrm{c}}$ in the $2 \mathrm{SC}$ phase, but such a description is ill defined beyond weak-coupling regimes.
} 

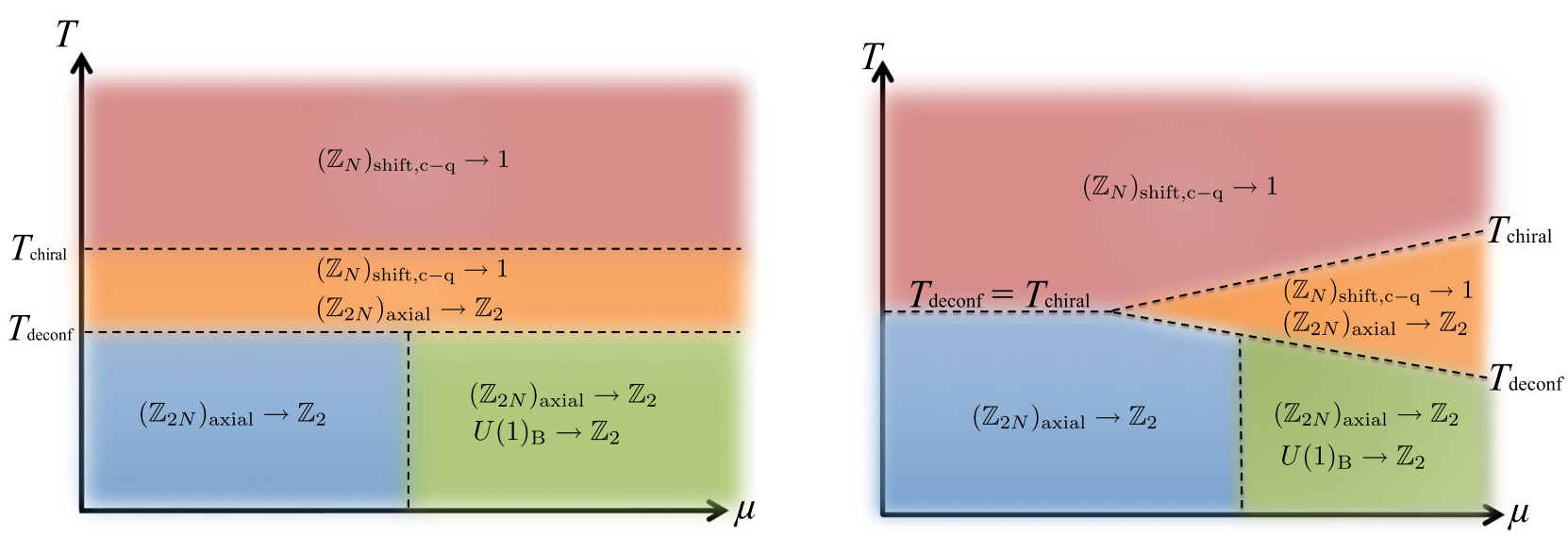

FIG. 1. Two examples of schematic $(T, \mu)$ phase diagrams of massless $\mathbb{Z}_{N}$-QCD $(N=3)$ that are consistent with the anomaly. Dashed lines show the phase transition lines, and the corresponding symmetry breaking pattern in each phase is specified. $T_{\text {chiral }}$ and $T_{\text {deconf }}$ denote the chiral restoration and deconfinement temperatures, respectively.

\section{CONCLUSION AND DISCUSSION}

In this paper, we for the first time put the anomalymatching constraint related to center and $\left(\mathbb{Z}_{2 N}\right)_{\text {axial }}$ symmetries on the finite- $(T, \mu)$ phase diagram of the $N$-flavor $S U(N)$ gauge theory under the $\mathbb{Z}_{N}$ flavor-twisted boundary condition. We first derived the mixed 't Hooft anomaly (9) of massless $N$-flavor QCD between vectorlike continuous symmetry and $\left(\mathbb{Z}_{2 N}\right)_{\text {axial }}$. Using this anomaly, we showed that massless $\mathbb{Z}_{N}$-QCD also has the 't Hooft anomaly (18) among $\left(\mathbb{Z}_{N}\right)_{\text {shift,c-q }}$, the maximal Abelian subgroup of vectorlike symmetry, and $\left(\mathbb{Z}_{2 N}\right)_{\text {axial }}$. The anomaly-matching argument shows that the phase diagram of massless $\mathbb{Z}_{N^{-}}$ QCD realizes a persistent order; i.e., the trivial phase cannot appear at any temperature $T$ and any quark chemical potential $\mu$. This is the rigorous result in $N$-flavor massless $\mathrm{QCD}$, and it can be found by considering the thermal expectation value of the operator given in (12).

As a consistency check, we compare this result with the known results about $\mathbb{Z}_{N}$-QCD. At low temperatures and low densities, the lattice simulation shows that discrete axial symmetry is spontaneously broken by the quark bilinear condensate. At sufficiently high temperatures and low densities, the one-loop effective potential of the Polyakov loop breaks the intertwined center symmetry $\left(\mathbb{Z}_{N}\right)_{\text {shift,c-q }}$, which is also confirmed by the lattice simulation. At low temperatures and sufficiently high densities, it is widely believed that the CFL phase appears, which breaks $U(1)_{\mathrm{B}}$ and $\left(\mathbb{Z}_{2 N}\right)_{\text {axial. }}$. The 2 SC or uSC phases are also observed in the effective model analysis, which breaks $\left(\mathbb{Z}_{N}\right)_{\text {shift,c-q }}$, as well as $\left(\mathbb{Z}_{2 N}\right)_{\text {axial }}$. In all these cases, SSB occurs so that the 't Hooft anomaly matching is satisfied. We further predict that $\mathbb{Z}_{N}$-QCD must have a nontrivial phase, such as SSB, conformal behavior, or topological order, even in the region where the system is strongly coupled and lattice simulation suffers from the sign problem. We present two examples of phase diagrams consistent with anomaly matching.
We would also like to make some speculative remarks on the $N$-flavor QCD phase diagram with the thermal boundary condition in the zero-temperature limit. Since our derivation of the anomaly has a four-dimensional origin, the anomaly-matching argument is valid however large the size $L$ of compactification is. By taking the zero-temperature limit, we expect that the effect of the boundary condition would disappear. If the vectorlike flavor symmetry is unbroken, it is indeed reasonable to argue that the effect of flavor dependence in the boundary condition disappears. Under this assumption, the anomaly-matching argument claims that finite-density massless $N$-flavor QCD shows a nontrivial phase at any quark chemical potential in the zero-temperature limit. This result also implies SSB of continuous axial symmetry since $\left(\mathbb{Z}_{2 N}\right)_{\text {axial }} \subset S U(N)_{\text {left }} \times$ $S U(N)_{\text {right }} \times U(1)_{\mathrm{q}}$. This gives the phenomenological impact on cold dense three-flavor massless QCD, because the possible chiral symmetry breaking is restricted even at finite densities $[67,68]$. We can check that this is indeed the case at least for small $\mu$ and also for sufficiently large $\mu$ in the zero-temperature limits, where the anomaly is satisfied by the SSB of discrete axial symmetry. At the zero temperature, anomaly matching for continuous chiral symmetry is also available [69,70], and we can obtain further constraints on possible dynamics in cold dense QCD.

\section{ACKNOWLEDGMENTS}

T. M. is grateful to H. Kouno for fruitful discussion. Y. K. and T.M. also thank the organizers of the workshop "Thermal Quantum Field Theory and Their Applications" at the Yukawa Institute of Theoretical Physics, Kyoto University. This work was started at the conference "RIMS-iTHEMS International Workshop on Resurgence Theory" at Kobe, Japan, and was completed during the workshop "Resurgent Asymptotics in Physics and Mathematics" at the Kavli Institute for Theoretical Physics. The authors greatly appreciate these opportunities 
and the hospitality of the organizers. Y. T. is financially supported by the RIKEN special postdoctoral program. Y. K. is supported by the Grants-in-Aid for JSPS fellows (Grant No. 15J01626). This work is supported in part by the Japan Society for the Promotion of Science (JSPS) Grant-in-Aid for Scientific Research (KAKENHI) Grant No. 16K17677 (T. M). T. M. and N. S. are also supported by the MEXT-Supported Program for the Strategic Research Foundation at Private Universities (Keio University)
"Topological Science" (Grant No. S1511006). Research at KITP is supported by the National Science Foundation under Grant No. NSF PHY-1125915.

Note added.-Recently, the authors were notified that a paper on S1-compactified QCD with some related aspects will appear [35]. The authors thank A. Cherman and M. Ünsal for sharing the unpublished manuscript.
[1] L. D. Landau, On the theory of phase transitions. I., Zh. Eksp. Teor. Fiz. 11, 19 (1937).

[2] V. L. Ginzburg and L. D. Landau, On the theory of superconductivity, Zh. Eksp. Teor. Fiz. 20, 35 (1950).

[3] G. 't Hooft, Naturalness, chiral symmetry, and spontaneous chiral symmetry breaking, in Recent Developments in Gauge Theories. Proceedings, Nato Advanced Study Institute, Cargese, France, 1979 (Springer, Boston, MA, 1980), Vol. 59, pp. 135-157, http://dx.doi.org/10.1007/9781-4684-7571-5_9.

[4] Y. Frishman, A. Schwimmer, T. Banks, and S. Yankielowicz, The axial anomaly and the bound state spectrum in confining theories, Nucl. Phys. B177, 157 (1981).

[5] S. R. Coleman and B. Grossman, 't Hooft's consistency condition as a consequence of analyticity and unitarity, Nucl. Phys. B203, 205 (1982).

[6] F. D. M. Haldane, Continuum dynamics of the 1-D Heisenberg antiferromagnetic identification with the $\mathrm{O}(3)$ nonlinear sigma model, Phys. Lett. 93A, 464 (1983).

[7] F. D. M. Haldane, Nonlinear Field Theory of Large Spin Heisenberg Antiferromagnets: Semiclassically Quantized Solitons of the One-Dimensional Easy Axis Neel State, Phys. Rev. Lett. 50, 1153 (1983).

[8] I. Affleck and E. H. Lieb, A proof of part of Haldane's conjecture on spin chains, Lett. Math. Phys. 12, 57 (1986).

[9] I. Affleck, T. Kennedy, E. H. Lieb, and H. Tasaki, Rigorous Results on Valence Bond Ground States in Antiferromagnets, Phys. Rev. Lett. 59, 799 (1987).

[10] F. D. M. Haldane, Model for a Quantum Hall Effect without Landau Levels: Condensed-Matter Realization of the "Parity Anomaly", Phys. Rev. Lett. 61, 2015 (1988).

[11] T. Senthil, Deconfined quantum critical points, Science 303, 1490 (2004).

[12] T. Senthil, L. Balents, S. Sachdev, A. Vishwanath, and M. P. A. Fisher, Quantum criticality beyond the Landau-GinzburgWilson paradigm, Phys. Rev. B 70, 144407 (2004).

[13] C. Wang, A. Nahum, M. A. Metlitski, C. Xu, and T. Senthil, Deconfined Quantum Critical Points: Symmetries and Dualities, Phys. Rev. X 7, 031051 (2017).

[14] A. Vishwanath and T. Senthil, Physics of Three Dimensional Bosonic Topological Insulators: Surface Deconfined Criticality and Quantized Magnetoelectric Effect, Phys. Rev. X 3, 011016 (2013).
[15] A. Kapustin and R. Thorngren, Anomalies of Discrete Symmetries in Three Dimensions and Group Cohomology, Phys. Rev. Lett. 112, 231602 (2014).

[16] A. Kapustin and R. Thorngren, Anomalies of discrete symmetries in various dimensions and group cohomology, arXiv:1404.3230.

[17] G. Y. Cho, J. C. Y. Teo, and S. Ryu, Conflicting symmetries in topologically ordered surface states of three-dimensional bosonic symmetry protected topological phases, Phys. Rev. B 89, 235103 (2014).

[18] J. C. Wang, Z.-C. Gu, and X.-G. Wen, Field Theory Representation of Gauge-Gravity Symmetry-Protected Topological Invariants, Group Cohomology and Beyond, Phys. Rev. Lett. 114, 031601 (2015).

[19] E. Witten, Fermion path integrals and topological phases, Rev. Mod. Phys. 88, 035001 (2016).

[20] N. Seiberg and E. Witten, Gapped boundary phases of topological insulators via weak coupling, Prog. Theor. Exp. Phys. 2016, 12C101 (2016).

[21] Y. Tachikawa and K. Yonekura, On time-reversal anomaly of $2+1 \mathrm{~d}$ topological phases, Prog. Theor. Exp. Phys. 2017, 033B04 (2017).

[22] Y. Tachikawa and K. Yonekura, More on Time-Reversal Anomaly of $2+1 \mathrm{~d}$ Topological Phases, Phys. Rev. Lett. 119, 111603 (2017).

[23] D. Gaiotto, A. Kapustin, Z. Komargodski, and N. Seiberg, Theta, Time Reversal, and Temperature, J. High Energy Phys. 05 (2017) 091.

[24] Y. Tanizaki and Y. Kikuchi, Vacuum structure of bifundamental gauge theories at finite topological angles, J. High Energy Phys. 06 (2017) 102.

[25] Z. Komargodski, A. Sharon, R. Thorngren, and X. Zhou, Comments on Abelian Higgs models and persistent order, arXiv:1705.04786.

[26] Z. Komargodski, T. Sulejmanpasic, and M. Ünsal, Walls, Anomalies, and (De)Confinement in Quantum AntiFerromagnets, arXiv:1706.05731.

[27] G. Y. Cho and S. Ryu, Anomaly manifestation of LiebSchultz-Mattis theorem and topological phases, Phys. Rev. B 96, 195105 (2017).

[28] H. Shimizu and K. Yonekura, Anomaly constraints on deconfinement and chiral phase transition, arXiv: 1706.06104. 
[29] J. Wang, X.-G. Wen, and E. Witten, Symmetric gapped interfaces of SPT and SET states: Systematic constructions, arXiv: 1705.06728.

[30] M. A. Metlitski and R. Thorngren, Intrinsic and emergent anomalies at deconfined critical points, arXiv:1707.07686.

[31] Y. Kikuchi and Y. Tanizaki, Global inconsistency, 't Hooft anomaly, and level crossing in quantum mechanics, Prog. Theor. Exp. Phys. 2017, 113B05 (2017).

[32] D. Gaiotto, Z. Komargodski, and N. Seiberg, Time-reversal breaking in $\mathrm{QCD}_{4}$, walls, and dualities in $2+1$ dimensions, J. High Energy Phys. 01 (2018) 110.

[33] Y. Tanizaki, T. Misumi, and N. Sakai, Circle compactification and 't Hooft anomaly, J. High Energy Phys. 12 (2017) 056.

[34] M. Yamazaki, Relating 't Hooft anomalies of 4d pure YangMills and 2d $\mathbb{C} \mathbb{P}^{N-1}$ model, arXiv:1711.04360.

[35] A. Cherman and M. Unsal, Critical behavior of gauge theories and Coulomb gases in three and four dimensions, arXiv:1711.10567.

[36] M. Guo, P. Putrov, and J. Wang, Time reversal, SU(N) Yang-Mills and cobordisms: Interacting topological superconductors/insulators and quantum spin liquids in $3+1 \mathrm{D}$, arXiv:1711.11587.

[37] M. G. Alford, A. Schmitt, K. Rajagopal, and T. Schafer, Color superconductivity in dense quark matter, Rev. Mod. Phys. 80, 1455 (2008).

[38] K. Fukushima and T. Hatsuda, The phase diagram of dense QCD, Rep. Prog. Phys. 74, 014001 (2011).

[39] K. Masuda, T. Hatsuda, and T. Takatsuka, Hadron-Quark crossover and massive hybrid stars with strangeness, Astrophys. J. 764, 12 (2013).

[40] I. M. Barbour, S. E. Morrison, E. G. Klepfish, J. B. Kogut, and M.-P. Lombardo, Results on finite density QCD, Nucl. Phys. B, Proc. Suppl. 60, 220 (1998).

[41] F. Karsch, Lattice QCD at high temperature and density, Lect. Notes Phys. 583, 209 (2002).

[42] S. Muroya, A. Nakamura, C. Nonaka, and T. Takaishi, Lattice QCD at finite density: An introductory review, Prog. Theor. Phys. 110, 615 (2003).

[43] T. D. Cohen, Functional Integrals for QCD at Nonzero Chemical Potential and Zero Density, Phys. Rev. Lett. 91, 222001 (2003).

[44] Y. Tanizaki, Y. Hidaka, and T. Hayata, Lefschetz-thimble analysis of the sign problem in one-site fermion model, New J. Phys. 18, 033002 (2016).

[45] H. Kouno, Y. Sakai, T. Makiyama, K. Tokunaga, T. Sasaki, and M. Yahiro, Quark-gluon thermodynamics with the $Z(N(c))$ symmetry, J. Phys. G 39, 085010 (2012).

[46] Y. Sakai, H. Kouno, T. Sasaki, and M. Yahiro, The quarkyonic phase and the $\mathrm{Z}_{N_{c}}$ symmetry, Phys. Lett. B 718, 130 (2012).

[47] H. Kouno, T. Makiyama, T. Sasaki, Y. Sakai, and M. Yahiro, Confinement and $\mathbb{Z}_{3}$ symmetry in three-flavor QCD, J. Phys. G 40, 095003 (2013).

[48] H. Kouno, T. Misumi, K. Kashiwa, T. Makiyama, T. Sasaki, and M. Yahiro, Differences and similarities between fundamental and adjoint matters in $\mathrm{SU}(\mathrm{N})$ gauge theories, Phys. Rev. D 88, 016002 (2013).

[49] E. Poppitz and T. Sulejmanpasic, (S)QCD on $\mathbb{R}^{3} \times \mathbb{S}^{1}$ : Screening of Polyakov loop by fundamental quarks and the demise of semi-classics, J. High Energy Phys. 09 (2013) 128.
[50] T. Iritani, E. Itou, and T. Misumi, Lattice study on QCD-like theory with exact center symmetry, J. High Energy Phys. 11 (2015) 159.

[51] H. Kouno, K. Kashiwa, J. Takahashi, T. Misumi, and M. Yahiro, Understanding QCD at high density from a $\mathrm{Z}_{3}$-symmetric QCD-like theory, Phys. Rev. D 93, 056009 (2016).

[52] T. Hirakida, H. Kouno, J. Takahashi, and M. Yahiro, Interplay between sign problem and $Z_{3}$ symmetry in threedimensional Potts models, Phys. Rev. D 94, 014011 (2016).

[53] T. Hirakida, J. Sugano, H. Kouno, J. Takahashi, and M. Yahiro, Sign problem in $Z_{3}$-symmetric effective Polyakovline model, Phys. Rev. D 96, 074031 (2017).

[54] A. Cherman, S. Sen, M. Unsal, M. L. Wagman, and L. G. Yaffe, Order Parameters and Color-Flavor Center Symmetry in QCD, Phys. Rev. Lett. 119, 222001 (2017).

[55] K. Fujikawa, Path Integral Measure for Gauge Invariant Fermion Theories, Phys. Rev. Lett. 42, 1195 (1979).

[56] K. Fujikawa, Path Integral for Gauge Theories with Fermions, Phys. Rev. D 21, 2848 (1980); Erratum, Phys. Rev. D 22, 1499(E) (1980).

[57] D. Gaiotto, A. Kapustin, N. Seiberg, and B. Willett, Generalized global symmetries, J. High Energy Phys. 02 (2015) 172.

[58] A. Kapustin and N. Seiberg, Coupling a QFT to a TQFT and duality, J. High Energy Phys. 04 (2014) 001.

[59] G. V. Dunne and M. Unsal, Resurgence and trans-series in quantum field theory: The $\mathrm{CP}\left(N^{-1}\right)$ model, J. High Energy Phys. 11 (2012) 170.

[60] G. V. Dunne and M. Unsal, Continuity and resurgence: Towards a continuum definition of the $\mathbb{C P}\left(N^{-1}\right)$ model, Phys. Rev. D 87, 025015 (2013).

[61] T. Misumi, M. Nitta, and N. Sakai, Neutral bions in the $\mathbb{C} P^{N-1}$ model, J. High Energy Phys. 06 (2014) 164.

[62] T. Misumi, M. Nitta, and N. Sakai, Non-BPS exact solutions and their relation to bions in $\mathbb{C} P^{N-1}$ models, J. High Energy Phys. 05 (2016) 057.

[63] T. Sulejmanpasic, Global Symmetries, Volume Independence, and Continuity in Quantum Field Theories, Phys. Rev. Lett. 118, 011601 (2017).

[64] D. J. Gross, R. D. Pisarski, and L. G. Yaffe, QCD and instantons at finite temperature, Rev. Mod. Phys. 53, 43 (1981).

[65] N. Weiss, The effective potential for the order parameter of gauge theories at finite temperature, Phys. Rev. D 24, 475 (1981).

[66] A. Roberge and N. Weiss, Gauge theories with imaginary chemical potential and the phases of QCD, Nucl. Phys. B275, 734 (1986).

[67] J. Stern, Light quark masses and condensates in QCD, Lect. Notes Phys. 513, 26 (1998).

[68] I. I. Kogan, A. Kovner, and M. A. Shifman, Chiral symmetry breaking without bilinear condensates, unbroken axial $Z(N)$ symmetry, and exact QCD inequalities, Phys. Rev. D 59, 016001 (1998).

[69] F. Sannino, A note on anomaly matching for finite density QCD, Phys. Lett. B 480, 280 (2000).

[70] S. D. H. Hsu, F. Sannino, and M. Schwetz, Anomaly matching in gauge theories at finite matter density, Mod. Phys. Lett. A 16, 1871 (2001). 